\title{
Hidden Yangians in 2D Massive Current Algebras
}

\author{
Denis Bernard \\ Service de Physique Théorique de Saclay, ${ }^{\star}$ F-91191 Gif-sur-Yvette, France
}

Received July 26, 1990; in revised form August 29, 1990

\begin{abstract}
We define non-local conserved currents in massive current algebras in two dimensions. Our approach is algebraic and non-perturbative. The non-local currents give a quantum field realization of the Yangians. We show how the noncocommutativity of the Yangians is related to the non-locality of the currents. We discuss the implications of the existence of non-local conserved charges on the $S$-matrices.
\end{abstract}

\section{Introduction}

Conformal field theories describe two dimensional critical systems but (if there is no cross-over) scaling limits near criticality are described by massive theories. The ultraviolet fixed points of the massive theories are the critical CFT's we started with. Recently part of the algebraic CFT framework [1, 2] has been pushed to the massive theories. This has mainly consisted in the identification of integrable perturbations of conformal field theories and of their $S$-matrices [3], in the comparison of these perturbations with known integrable models and their restrictions [4-7], in the lift of CFT moduli diagrams to the moduli space of integrable models [8], etc.....

Moreover non-local conserved currents have been recognized as being hidden in massive integrable perturbations of CFT's [8-10]. On-shell they lead to algebraic equations which determine the factorizable $S$-matrices. There are two kinds of non-local conserved currents: i) Either the associated conserved charges have fractional Lorentz spins like in the (fractional supersymmetric) sine-Gordon models [8]. In these cases the factorizable $S$-matrices are trigonometric solutions of the quantum Yang-Baxter equations. ii) Or the non-local conserved charges have zero spin. In these cases the factorizable $S$-matrices are rational solutions of the Yang-Baxter equations.

* Laboratoire de la Direction des sciences de la matière du Commissariat à l'énergie atomique 
In this paper we describe the simplest cases: namely the cases of non-local conserved charges of zero spin. To be precise we will show that in massive current algebras there are hidden quantum non-local conserved currents which are offshell realizations of the Yangians [11]. In order to have an exact and nonperturbative approach we define the theories algebraically by imposing constraints on the operator algebras. This approach parallels what has been done in conformal field theories. The operator algebras we will be interested in are the massive current algebras. They describe perturbations of affine Kac-Moody algebras $[12,13]$. The non-local conserved currents are defined nonperturbatively in terms of the generators of the massive current algebras. They generate quantum non-commutative and non-cocommutative algebras. We show how the non-cocommutativity and the comultiplications are related to the nonlocality through the braiding relations satisfied by the non-local conserved currents. We illustrate how to deduce algebraic equations for the $S$-matrices using the non-local conserved charges. The successive steps involved in the construction are described in Sects. $2 \mathrm{a}-2 \mathrm{~h}$.

We stress that the main point of this paper is not that we found the Yangians but that non-local conserved currents can be non-perturbatively defined. This promotes them to a central position in the algebraic games, alternative to the quantum inverse scattering methods, which are involved in the two-dimensional integrable models. This paper is mainly dedicated to illustrate how to deal with non-local currents.

\section{Non-Local Conserved Currents in 2D Massive Current Algebras}

We show how an algebraic approach to the 2D massive current algebras allows us to deduce non-perturbative results. This statement is illustrated with the exact and non-perturbative construction of non-local conserved currents in massive current algebras. In order to clarify the logic of the approach we formulate the construction in eight successive steps labeled from (2a) to (2h).

\section{2a) The Classical Cases}

Before plunging into the quantum cases let us first describe the classical models we have in mind. These are two-dimensional geometrical models. They are dealing with a one-form, denoted by $J(x)$, valued in a semi-simple Lie algebra $\mathscr{G}$ : $J(x)=\sum_{a} J_{\mu}^{a}(x) t^{a} d x^{\mu}$, where $t^{a}, a=1, \ldots, \operatorname{dim} \mathscr{G}$, form a basis of $\mathscr{G}^{1}$. By definition of the classical models we assume that the equations of motion impose to $J(x)$ to be a curl-free conserved current:

$$
\begin{gathered}
d * J=0 \text { and } d J+[J, J]=0, \\
\partial_{\mu} J_{\mu}^{a}(x)=0 \text { and } \partial_{\mu} J_{v}^{a}(x)-\partial_{v} J_{\mu}^{a}(x)+f^{a b c} J_{\mu}^{b}(x) J_{v}^{c}(x)=0 .
\end{gathered}
$$

${ }^{1}$ We suppose the $t^{a}$ orthonormalized. We use the convention: $\left[t^{a}, t^{b}\right]=f^{a b c} t^{c}$, where $f^{a b c}$ denote the structure constants of $\mathscr{G}$ 
The equations of motion (1) admit a Lax representation. Namely they are equivalent to the zero curvature condition, $\left[\mathscr{D}_{\mu}(\lambda), \mathscr{D}_{v}(\lambda)\right]=0$, for the connection $\mathscr{D}_{\mu}(\lambda)$,

$$
\mathscr{D}_{\mu}(\lambda)=\partial_{\mu}+\frac{\lambda^{2}}{\lambda^{2}-1} J_{\mu}(x)+\frac{\lambda}{\lambda^{2}-1} \varepsilon_{\mu v} J_{v}(x) .
$$

The hamiltonian structures for these models have been discussed in [14].

The geometrical character of Eq. (1) leads to the definition of an infinite set of conserved charges. We stress that these conserved charges are non-local. They can be defined in two different but equivalent ways: i) either one uses the transfer matrix defined from the connection $\mathscr{D}_{\mu}(\lambda)$ as a generating function for the non-local conserved charges as in [15], ii) or one uses a recursive definition of the conserved currents as explained in [16]. In the following we only need the two first conserved currents. The first one is the local one-form $J^{(0) a}(x)=J_{\mu}^{a}(x)$. The second one, denoted by $J^{(1)}(x)=\sum_{a} J_{\mu}^{(1) a}(x) t^{a} d x^{\mu}$, is defined by:

$$
\begin{gathered}
J^{(1)}=* J+\frac{1}{2}[J, \phi] \quad \text { with } d \phi=* J, \\
J_{\mu}^{(1) a}(x)=\varepsilon_{\mu \nu} J_{v}^{a}(x)+\frac{1}{2} f^{a b c} J_{\mu}^{b}(x) \phi^{c}(x) \quad \text { with } \partial_{\mu} \phi^{c}(x)=\varepsilon_{\mu v} J_{v}^{c}(x) .
\end{gathered}
$$

The currents $J_{\mu}^{(1) a}(x)$ are non-local because $\phi^{c}(x)$ is non-local: $\phi^{c}(x)=\int_{\mathscr{C}_{x}} * J^{c}$, where $\mathscr{C}_{x}$ is a curve ending at the point $x$. Using Eq. (1) it is easy to check that the currents $J^{(1) a}(x)$ are conserved: $\partial_{\mu} J_{\mu}^{(1) a}(x)=0$. The currents $J_{\mu}^{(1) a}(x)$ are the currents we want to quantize.

\section{2b) The Massive Current Algebras}

First let us make few comments about the general framework in which the massive current algebras fit. A massive QFT is characterized by its set of operators $\theta_{\alpha}(x)$ which close by OPE: $\mathcal{O}_{\alpha}(x) \mathcal{O}_{\beta}(0) \sim \sum_{\gamma} C_{\alpha \beta}^{\gamma}(x) \mathcal{O}_{\gamma}(0)$. The operators $\mathcal{O}_{\alpha}(x)$ are defined by their sets of correlation functions ${ }^{2}$. The ultraviolet limit of a massive QFT is a conformal field theory. The two basic hypotheses that we are going to make are the following:

-) All the operators $\mathcal{O}_{\alpha}(x)$ have a smooth ultraviolet limit which we denote by $\mathcal{O}_{\alpha}^{*}(x)$;

-.) the correspondence between the operators $\mathcal{O}_{\alpha}(x)$ and $\mathcal{O}_{\alpha}^{*}(x)$ is one-to-one. These hypothesis are implicit in the approach to massive QFT from perturbed CFT as advocated by Zamolodchikov [3]. In practice these hypotheses allow us to label the fields of the massive theory by the fields of the ultraviolet CFT. Moreover the fields $\mathcal{O}_{\alpha}(x)$ of the massive theory have the same scaling dimensions and the same spins as the fields $\mathcal{O}_{\alpha}^{*}(x)$ of the ultraviolet CFT. Note that not all the massive theories fulfilled these two properties. Standard counter-examples are free massive bosons or $O(n)$ models. In these cases the two properties are spoiled by infrared divergences and the perturbing fields are not fields of the ultraviolet CFT's.

Having these preliminary remarks in mind we can now attempt to define the massive current algebras.

${ }^{2}$ Because we do not gain more information by adding derivatives of the correlation functions we understand the data of the operators $\mathcal{O}_{\alpha}(x)$ as equivalent to the data of all their multi-derivatives $\partial_{\mu_{1}} \ldots \partial_{\mu_{P}} \mathcal{O}_{\alpha}(x)$ 
Definition. A massive current algebra is an operator algebra (in a Lorentz and PT covariant quantum field theory) generated by an operator one-form $J(x)=\sum_{a} J_{\mu}^{a}(x) t^{a} d x^{\mu}$ valued in a semi-simple Lie algebra $\mathscr{G}$ which satisfy the following properties:

(i) The currents $J_{\mu}^{a}(x)$ are local and conserved: $\partial_{\mu} J_{\mu}^{a}(x)=0$.

(ii) The ultraviolet limit is a smooth limit. Moreover the ultraviolet limit of the currents $J_{\mu}^{a}(x)$ are $\mathscr{G}^{(1)}$ Kac-Moody currents.

(iii) The OPE's of the currents $J_{\mu}^{a}(x) J_{\mu}^{b}(x)$ close only on the $\mathscr{G}^{(1)}$ current-module. I.e. the OPE's of the currents only involve fields $\mathcal{O}_{\alpha}(x)$ (and their multi-derivatives) whose ultraviolet limit are fields in the $\mathscr{G}^{(1)}[1] \otimes[1]$-module.

We believe that these conditions are enough to characterize a massive current algebra. But obviously this statement needs further studies. However in the following we will use hypotheses weaker than these three conditions. This implies that part of our results will remain valid in more general contexts.

A hidden consequence of this definition is that the massive current algebras actually describe perturbations of $\mathscr{G}^{(1)}$ affine Kac-Moody algebras by the perturbing fields

$$
\Phi_{\text {pert. }}(x)=\sum_{a} J_{\mu}^{a}(x) J_{\mu}^{a}(x) .
$$

In any $\mathscr{G}$-invariant perturbations of affine Kac-Moody algebras, an operator algebra generated by a $\mathscr{G}$-valued conserved current will exist. However we prefer to keep the name "massive current algebra" for the definition we gave above because in the other cases the OPE's of the currents will mix the $\mathscr{G}^{(1)}$ current-module with the other $\mathscr{G}^{(1)}$ modules.

\section{2c) Lüscher's Theorem and Its Applications}

In this subsection we show that the quantum currents $J_{\mu}^{a}(x)$ generating a massive current algebra are curl-free:

$$
\partial_{\mu} J_{v}^{a}(x)-\partial_{v} J_{\mu}^{a}(x)+f^{a b c}: J_{\mu}^{b}(x) J_{v}^{c}(x):=0,
$$

where the double dots denote an appropriate normal order. The proof of Eq. (4) relies on a slight extension of theorem due to Lüscher [17].

Lüscher's theorem describes the OPE's between currents in a massive current algebra. Lüscher found it while studying the $O(n)$ models which do not satisfy the conditions exposed in Sect. 2b. However, as we will explain, part of its results remains valid in our approach. Lüscher's theorem can be stated in the following way ${ }^{3}$ :

Theorem 1 (Lüscher). Let $J_{\mu}^{a}(x)$ be the conserved currents generating a massive current algebra. Suppose that the conditions (i) and (iii) of Sect. $2 b$ are fulfilled. Then we have the following OPE's for $x^{2}<0$ :

$$
\begin{aligned}
& f^{a b c} J_{\mu}^{b}(x) J_{v}^{c}(0) \\
&=\left(C_{1} x^{2} \eta_{\mu v} x^{\varrho}+C_{2} x^{2}\left(x_{\mu} \delta_{v}^{\varrho}+x_{v} \delta_{\mu}^{\varrho}\right)+C_{3} x_{\mu} x_{v} x^{\varrho}\right)\left(J_{\varrho}^{a}(0)+\frac{1}{2} x^{\sigma} \partial_{\sigma} J_{\varrho}^{a}(0)\right) \\
&+\left(D_{1} x^{\varrho}\left(x_{\mu} \delta_{v}^{\sigma}-x_{v} \delta_{\mu}^{\sigma}\right)+D_{2} x^{\sigma}\left(x_{\mu} \delta_{v}^{\varrho}-x_{v} \delta_{\mu}^{\varrho}\right)\right)\left(\partial_{\sigma} J_{\varrho}^{a}(0)\right) \\
&+\mathcal{O}\left(|x|^{1.0}\right) .
\end{aligned}
$$

3 We used the following space-time conventions $x^{\nu} \equiv\left(x^{0}=t, x^{1}=x\right) ; \quad x^{ \pm}=x \pm t ;$ and $d s^{2}=\eta_{\mu v} d x^{\mu} d x^{v}=d t^{2}-d x^{2}$ 
The coefficients $C_{i}$ and $D_{i}$ only depend on $x^{2}$. Furthermore they satisfy the following differential equations:

and

$$
\begin{gathered}
x^{2} \frac{d}{d x^{2}} C_{2}=-\frac{1}{2}\left(C_{1}+5 C_{2}\right), \\
x^{2} \frac{d}{d x^{2}}\left(C_{1}+C_{2}+C_{3}\right)=-\left(C_{1}+C_{2}+2 C_{3}\right),
\end{gathered}
$$

$$
\begin{aligned}
& x^{2} \frac{d}{d x^{2}} D_{1}=-D_{1}-\frac{x^{2}}{4} C_{1}, \\
& x^{2} \frac{d}{d x^{2}} D_{2}=-D_{2}-\frac{x^{2}}{4} C_{2}, \\
& x^{2} \frac{d}{d x^{2}}\left(D_{1}+D_{2}\right)=\frac{x^{2}}{4} C_{3} .
\end{aligned}
$$

We just sketch the proof. By hypothesis (iii) the left-hand side of (5) decomposes on fields which are multi-derivatives of fields whose ultraviolet limit are fields in the $\mathscr{G}^{(1)}[1] \otimes[1]$-module. Moreover because the currents have scaling dimension one and because we are evaluating the OPE up to order $\mathcal{O}\left(|x|^{1-0}\right)$ we are looking for fields having dimension zero, one or two. Global $\mathscr{G}$-invariance imposes to these fields to belong to the adjoint representation of $\mathscr{G}$. The only fields satisfying all these requirements are $J_{\varrho}^{a}(0)$ and $\partial_{\sigma} J_{\varrho}^{a}(0)$. Thus in a massive current algebra we have ${ }^{4}$ :

$$
f^{a b c} J_{\mu}^{b}(x) J_{v}^{c}(0)=\mathscr{C}_{\mu \nu}^{\varrho}(x) J_{\varrho}^{a}(0)+\mathscr{D}_{\mu \nu}^{\sigma e}(x)\left(\partial_{\sigma} J_{\varrho}^{a}(0)\right)+\mathcal{O}\left(|x|^{1-0}\right) .
$$

The rest of the proof is as in Lüscher's paper. The conservation law for $J_{\mu}^{a}$ allows us to choose $\mathscr{D}_{\mu \nu}^{\sigma e}(x)$ to be traceless: $\eta_{\sigma Q} \mathscr{D}_{\mu \nu}^{\sigma e}(x)=0$. Locality, PT-invariance and Lorentz covariance determine $\mathscr{C}_{\mu \nu}^{e}(x)$ and $\mathscr{D}_{\mu \nu}^{\sigma e}(x)$ as in Eq. (5). The conservation law for the currents implies the differential Eqs. (6) and (7).

The differential Eqs. (6) and (7) do not specify uniquely the unknown coefficients $C_{i}\left(x^{2}\right)$ and $D_{i}\left(x^{2}\right)$. But we have the following theorem ${ }^{5}$ :

Theorem 2. Let $J_{\mu}^{a}(x)$ be as in Theorem 1. Suppose moreover that the U.V. limit is smooth (hypothesis (ii) of Sect.(2b)), then the leading behavior of the functions $C_{i}\left(x^{2}\right)$ and $D_{i}\left(x^{2}\right)$ are:

$$
\begin{gathered}
C_{1}\left(x^{2}\right)=-C_{2}\left(x^{2}\right)=-\frac{\lambda}{\left(x^{2}\right)^{2}}+\mathcal{O}\left(|x|^{-3-0}\right), \\
C_{3}\left(x^{2}\right)=0+\mathcal{O}\left(|x|^{-3-0}\right), \\
D_{1}\left(x^{2}\right)=-D_{2}\left(x^{2}\right)=\frac{\lambda}{4 x^{2}} \log \left(-M^{2} x^{2}\right)+\mathcal{O}\left(|x|^{-1-0}\right),
\end{gathered}
$$

where $\lambda$ is an (uninteresting) constant depending on the normalization of the currents and $M$ is a (meaningful) constant related to the mass scale.

${ }^{4}$ Note that we used hypothesis weaker than hypothesis (iii) of Sect. $2 b$

5 At this point we differ from Lüscher because, as we already said, he was considering the $O(n)$ models whose U.V. limits are not smooth 
The proof of the Theorem 2 goes as follows. The smoothness of the U.V. limit implies the chiral splitting of the leading terms of the OPE's (8). This translates into the constraints: $\mathscr{C}_{+-}^{a}=\mathscr{C}_{++}^{-}=\mathscr{C}_{-}^{+}=0$. In their turn they imply the following algebraic relations among the coefficients $C_{i}\left(x^{2}\right)$ : $C_{1}+C_{2}=C_{3}=0$. These relations together with the differential equations (6) completely determine the functions $C_{i}\left(x^{2}\right)$. The functions $D_{i}\left(x^{2}\right)$ are then unambiguously defined (up to the mass scale parameter) by the differential equations (7).

Other current OPE's can be deduced using the same techniques. It is worthwhile to write explicitly the OPE's (5) while taking the relations (9) into account:

$$
\begin{gathered}
f^{a b c} J_{ \pm}^{b}(x) J_{ \pm}^{c}(0)=\frac{\lambda}{x^{ \pm}} J_{ \pm}^{a}(0)+\mathcal{O}\left(|x|^{-0}\right), \\
\frac{1}{2} f^{a b c}\left(J_{+}^{b}(x) J_{-}^{c}(0)-J_{-}^{b}(x) J_{+}^{c}(0)\right) \\
=-\frac{\lambda}{4} \log \left(M^{2} x^{+} x^{-}\right)\left(\partial_{+} J_{-}^{a}(0)-\partial_{-} J_{+}^{a}(0)\right)+\mathcal{O}\left(|x|^{1-0}\right) .
\end{gathered}
$$

The curl-free equation (4) is an immediate consequence of Eq.(10b). The normal order in (4) is defined in such a way to cancel the logarithmic divergence in $f^{a b c} J_{\mu}^{b} J_{v}^{c}$.

Recall that, as we said above, the massive current algebras are actually perturbations of $\mathscr{G}^{(1)}$ affine Kac-Moody algebras by the perturbing fields $\Phi_{\text {pert. }}(x)$ $=\sum_{a} J_{\mu}^{a}(x) J_{\mu}^{a}(x)$. Thus massive current algebras are also characterized by the level $K$ of the affine Kac-Moody algebras. However the OPE's (10) and the curl-free equation (4) are model independent in the sense that they do not depend on the level.

\section{2d) Quantum Definition of the Non-Local Conserved Currents}

Having proved that the quantum conserved currents satisfy the quantum form (4) of the equations of motion (1), it is now easy to define the quantum conserved currents $J^{(1)}(x, t)$. We define them by a point splitting regularization $(\delta>0)$ :

$$
\begin{gathered}
J_{\mu}^{(1) a}(x, t)=\lim _{\delta \rightarrow 0^{+}} J_{\mu}^{(1) a}(x, t \mid \delta), \\
J_{\mu}^{(1) a}(x, t \mid \delta)=Z(\delta) \varepsilon_{\mu v} J_{v}^{a}(x, t)+\frac{1}{2} f^{a b c} J_{\mu}^{b}(x, t) \phi^{c}(x-\delta, t),
\end{gathered}
$$

where $\phi^{c}(x, t)$, which satisfies $d \phi^{c}=* J^{c}$, is defined by:

$$
\phi^{c}(x, t)=\int_{\mathscr{C}_{x}} * J^{c} .
$$

The contour of integration $\mathscr{C}_{x}$ is a curve from $-\infty$ to $x$ with the convention drawn in Fig. 1. $\phi(x, t)$ depends weakly on the contour $\mathscr{C}_{x}$ because $* J$ is closed: $d * J=0$.

In Eq. (11) $Z(\delta)$ is a renormalization constant that we now determine by requiring that $J_{\mu}^{(1) a}(x, t)$ are finite and conserved.

Proposition. The non-local currents $J_{\mu}^{(1) a}(x, t)$ are finite and conserved $\left(\partial_{\mu} J_{\mu}^{(1) a}=0\right)$ if and only if

$$
Z(\delta)=\frac{\lambda}{2} \log (M \delta)+\mathcal{O}\left(|\delta|^{1-0}\right) .
$$




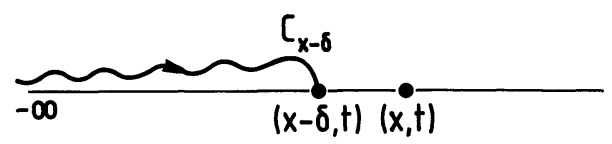

Fig. 1. Position of the string $\mathscr{C}_{x}$ in the definition (11) of the non-local currents

The proof is the following. First it is easily seen from Eq. $(10 \mathrm{~b})$ that $J_{\mu}^{(1) a}(x, t)$ is finite whenever $Z(\delta)=\frac{\lambda}{2} \log (\delta)+$ constant. The constant is fixed by demanding the conservation law for $J_{\mu}^{(1) a}$. [The other subleading terms in $Z(\delta)$ are meaningless.] Using Eq. (12) we deduce,

$$
\partial_{\mu} J_{\mu}^{(1) a}(x, t \mid \delta)=\frac{1}{2} \varepsilon_{\mu \nu}\left[Z(\delta)\left(\partial_{\mu} J_{v}^{a}-\partial_{v} J_{\mu}^{a}\right)(x, t)+f^{a b c} J_{\mu}^{b}(x, t) J_{v}^{c}(x-\delta, t)\right] .
$$

From Eq. (10b) we learn that $\partial_{\mu} J_{\mu}^{(1) a}(x, t \mid \delta)$ vanishes when $\delta \rightarrow 0$ if $Z(\delta)=\frac{\lambda}{2} \log (M \delta)$.

\section{2e) The Braiding Relations}

The non-local character of the currents $J^{(1)}(x, t)$ is encoded in their braiding relations. The latter are described by the following proposition.

Proposition. Let $\Phi(y, t)$ be a quantum field local with respect to the currents $J_{\mu}^{a}(x, t)$. Then it satisfies the following equal-time braiding relations:

$$
\begin{gathered}
J_{\mu}^{(1) a}(x, t) \Phi(y, t)=\Phi(y, t) J_{\mu}^{(1) a}(x, t) ; \quad \text { for } \quad x<y \\
J_{\mu}^{(1) a}(x, t) \Phi(y, t)=\Phi(y, t) J_{\mu}^{(1) a}(x, t)-\frac{1}{2} f^{a b c} Q_{0}^{b}(\Phi(y, t)) J_{\mu}^{c}(x, t) ; \quad \text { for } \quad x>y,
\end{gathered}
$$

where $Q_{0}^{b}$ are the global charges associated with the local conserved current $J_{\mu}^{b}$. (See the more precise definition in Eq. (19) below.)

We recall that the locality of $\Phi(y, t)$ with respect to $J_{\mu}^{a}(x, t)$ means that

$$
J_{\mu}^{a}(x, t) \Phi(y, t)=\Phi(y, t) J_{\mu}^{a}(x, t) ; \text { for } x \lessgtr y .
$$

The proof of the braiding relations (15) is the same as the proof of the braiding relations for disorder fields. It only relies on the way to deform the contour $\mathscr{C}_{x}$ entering in the definition of the currents $J^{(1)}(x, t)^{6}$. The relative positions of the contours $\mathscr{C}_{x}$ depend if $J^{(1)}(x, t)$ acts first or second. (Remember that product of operators are defined by time ordering.) Let us first consider the relation (15a). The position of the contours relative to the left-hand side and to the right-hand side of Eq. (15a) are drawn in Figs. $2 a$ and 2 b. One sees that there is no topological

${ }^{6}$ Braiding relations are purely topological. So we don't bother to keep the cumbersome notation due to the point-splitting regularization. It may easily be seen that the point-splitting procedure does not modify the braiding relations 


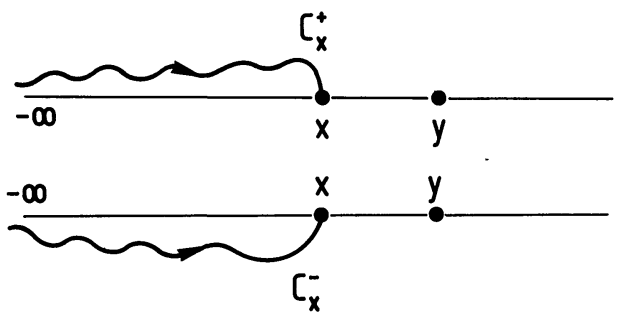

Fig. 2. a Position of the string $\mathscr{C}_{x}$ in the product $J^{(1)}(x, t) \Phi_{\Lambda}(y, t)$ with $x<y$. b Position of the string $\mathscr{C}_{x}$ in the product $\Phi_{A}(y, t) J^{(1)}(x, t)$ with $x<y$

obstruction for moving the contour from the configuration $\mathscr{C}_{x}^{+}$(Fig. 2a) to the configuration $\mathscr{C}_{x}^{-}$(Fig. 2b). This proves the relation (15a).

Let us now consider the relation (15b). The different configurations for the contours $\mathscr{C}_{x}$ are drawn in Fig. 3. One sees that there is an obstruction for moving the contour $\mathscr{C}_{x}^{+}$, Fig. 3a, onto the contour $\mathscr{C}_{x}^{-}$, Fig. 3b. This implies non-trivial braiding relations. All the non-locality of the currents $J^{(1) a}(x, t)$ is concentrated in the fields $\phi^{c}(x, t)$, Eq. (12). For $x>y$ the exchange relation between $\phi^{c}(x, t)$ and $\Phi(y, t)$ is:

$$
\begin{aligned}
\phi^{c}(x, t) \Phi(y, t) & =\int_{z \in \mathscr{C}_{x}^{+}} * J^{c}(z) \Phi(y, t)=\int_{z \in \gamma(y)} * J^{c}(z) \Phi(y, t)+\int_{z \in \mathscr{C}_{\bar{x}}} * J^{c}(z) \Phi(y, t) \\
& =Q_{0}^{c}(\Phi(y, t))+\Phi(y, t) \phi^{c}(x, t) .
\end{aligned}
$$

In the last equation we have used the definition of the global isotopic charge $Q_{0}^{c}$ [Eq. (19) below] and the fact that the fields $J_{\mu}^{c}$ and $\Phi$ are local. Plugging back Eq. (17) into the definition of the non-local current $J^{(1) a}$ and using once more the locality between the currents $J_{\mu}^{a}$ and the field $\Phi$ proves the braiding relations $(15 \mathrm{~b})$.

\section{2f) The (Non-Local) Conserved Charges}

Here we give the definition of the conserved charges. We make the distinction between charges acting on the states and on the fields ${ }^{7}$. We show how ones are graded (in a generalized sense) commutators of the other ones. We derive the complication from the braiding relations.

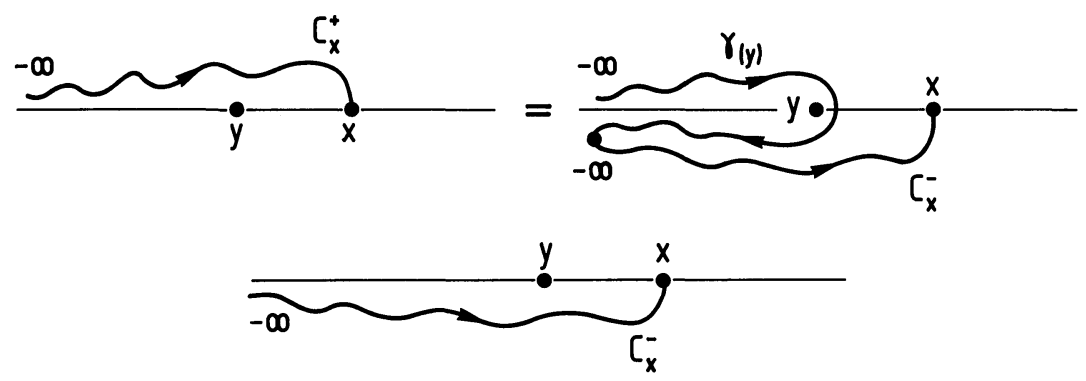

Fig. 3. a Position of the string $\mathscr{C}_{x}$ in the product $J^{(1)}(x, t) \Phi_{\Lambda}(y, t)$ with $x>y$. b Position of the string $\mathscr{C}_{x}$ in the product $\Phi_{A}(y, t) J^{(1)}(x, t)$ with $x>y$

${ }^{7}$ I thank G. Felder for his help in clarifying these points 


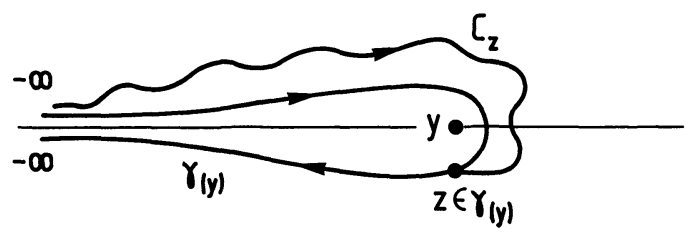

Fig. 4. Contours used in the definition (19) of the charges

Given conserved currents the associated charges are defined by integrating their dual forms along some curves. The charges depend weakly on the contours of integration because the dual forms are closed. The global conserved charges acting on the states of the physical Hilbert space are defined by choosing the domain of integration to be an equal-time slice. Namely for the currents $J_{\mu}^{(k) a}(x, t)$ :

$$
Q_{k}^{a}=\int_{t=c s t} d x J_{\mu}^{(k) a}(x, t) .
$$

The charges acting on a field $\Phi(y)$ located at a point $y$ are defined by choosing the contour of integration $\gamma(y)$ from $-\infty$ to $-\infty$ but surrounding the point $y$ :

$$
Q_{k}^{a}(\Phi(y))=\int_{z \in \gamma(y)} * J^{(k)^{a}}(z) \Phi(y) .
$$

The contour $\gamma(y)$ is drawn in Fig. 4. There we have drawn the position of the string $\mathscr{C}_{z}$ used in the definition of $J^{(1)}(z)$.

When the field $\Phi(y)$ is local with respect to the conserved currents, the contour $\gamma(y)$ can be closed and deformed into a small contour surrounding the point $y$. In this case the integral (19) picks up the residue of the OPE between the currents and the field. This applies to the currents $J_{\mu}^{a}(x)$ and to the charges $Q_{0}^{a}$. Moreover deforming the contour $\gamma(y)$ proves that

$$
Q_{0}^{a}(\Phi(y))=Q_{0}^{a} \Phi(y)-\Phi(y) Q_{0}^{a} .
$$

When the currents and the field $\Phi(y)$ are not respectively local the situation is more subtle. The contour $\gamma(y)$ can no more be closed and the action of the charges on the field is no more a pure commutator. For the non-local conserved currents $J^{(1)}(x)$ the relation between the global charges (18) acting on the states and the charges (19) acting on the fields is the following:

$$
Q_{1}^{a}(\Phi(y))=Q_{1}^{a} \Phi(y)-\Phi(y) Q_{1}^{a}+\frac{1}{2} f^{a b c} Q_{0}^{b}(\Phi(y)) Q_{0}^{c} .
$$

The proof of Eq. (21) consists in decomposing the contour of integration $\gamma(y)$ into the difference of two contours $\gamma_{+}$and $\gamma_{-}$as shown in Fig. 5, and in using the braiding relation (15b) when the current $J^{b}(x)$ is on $\gamma_{-}$.

Let us now describe the comultiplications. We denote them by $\Delta$. The comultiplications just encode how the charges act on a product of fields, say $\Phi_{1}\left(y_{1}\right) \Phi_{2}\left(y_{2}\right) \ldots$. In the case of the charges $Q_{0}^{a}$ and for fields $\Phi_{n}\left(y_{n}\right)$ which are local with respect to the currents $J_{\mu}^{a}(x)$ all the contours can be deformed without trouble and we have:

$$
Q_{0}^{a}\left(\Phi_{1}\left(y_{1}\right) \Phi_{2}\left(y_{2}\right)\right)=Q_{0}^{a}\left(\Phi_{1}\left(y_{1}\right)\right) \Phi_{2}\left(y_{2}\right)+\Phi_{1}\left(y_{1}\right) Q_{0}^{a}\left(\Phi_{2}\left(y_{2}\right)\right)
$$

or equivalently,

$$
\Delta Q_{0}^{a}=Q_{0}^{a} \otimes 1+1 \otimes Q_{0}^{a} .
$$

It is the standard Lie algebra comultiplication as it should be. 


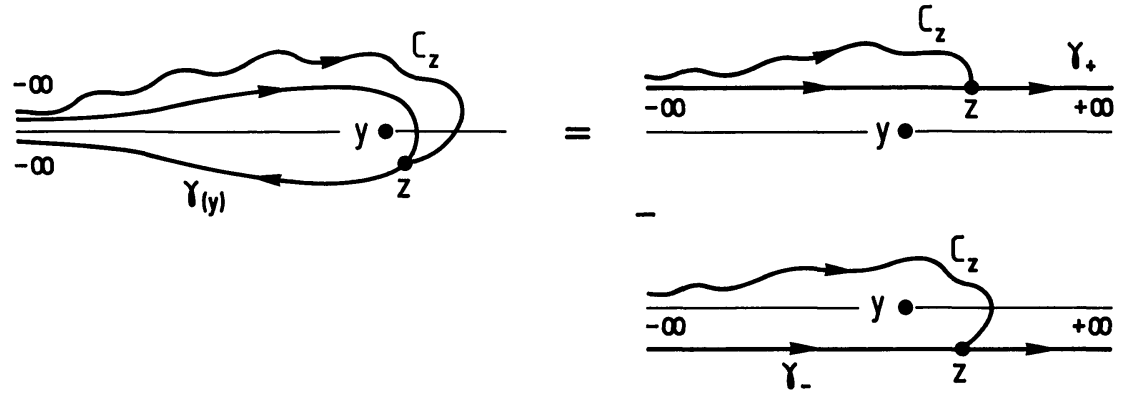

Fig. 5. Decomposition of the contours used in relating the actions of the charges to graded commutators

In the case of the non-local charges $Q_{1}^{a}$ the standard comultiplication is deformed due to the non-trivial braiding relations between the non-local currents and the fields.

Proposition. Let $\Phi_{n}\left(y_{n}\right)$ be quantum fields local with respect to the currents $J_{\mu}^{a}(x)$. Then we have the following comultiplication for the non-local conserved charges $Q_{1}^{a}$ :

$$
\begin{aligned}
Q_{1}^{a}\left(\Phi_{1}\left(y_{1}\right) \Phi_{2}\left(y_{2}\right)\right)= & Q_{1}^{a}\left(\Phi_{1}\left(y_{1}\right)\right) \Phi_{2}\left(y_{2}\right)+\Phi_{1}\left(y_{1}\right) Q_{1}^{a}\left(\Phi_{2}\left(y_{2}\right)\right) \\
& -\frac{1}{2} f^{a b c} Q_{0}^{b}\left(\Phi_{1}\left(y_{1}\right)\right) Q_{0}^{c}\left(\Phi_{2}\left(y_{2}\right)\right)
\end{aligned}
$$

or equivalently,

$$
\Delta Q_{1}^{a}=Q_{1}^{a} \otimes 1+1 \otimes Q_{1}^{a}-\frac{1}{2} f^{a b c} Q_{0}^{b} \otimes Q_{0}^{c} .
$$

Equation (23a) can be proved by decomposing the contour $\gamma_{12}$ used in defining the action of $Q_{1}^{a}$ on the product $\Phi_{1}\left(y_{1}\right) \Phi_{2}\left(y_{2}\right)$. The contour $\gamma_{12}$ is surrounding the two points $y_{1}$ and $y_{2}$. It decomposes into the sum of two contours $\gamma_{1}$ and $\gamma_{2}$ surrounding $y_{1}$ and $y_{2}$ respectively. But on the contour $\gamma_{2}$ we have to use the braiding relations (15) in order to pass the string $\mathscr{C}_{z}$ through the point $y_{1}$, see Fig. 6. Equation (23) can also be proved starting from the graded commutators (21).

\section{2g) The Algebra of the Conserved Charges}

Here we show that the (non-local) conserved charges generate a non-abelian extension of the two-dimensional Lorentz algebra. This is possible since the Coleman-Mandula theorem [18] breaks down in two dimensions because the comultiplication of the symmetry algebra could be non-trivial. In two dimensions the Poincare algebra which is generated by the momentum operators $P_{\mu}$ and the Lorentz boost $L$ is abelian. The momentum operators $P_{\mu}$ are the global charges associated with the conserved stress-tensor $T_{\mu v}(x): \partial_{\mu} T_{\mu v}(x)=0^{8}$. The Lorentz boost $L$ is the global charge associated with the conserved boost current:

$$
L_{\mu}(x)=\frac{1}{2} \varepsilon^{\varrho \sigma}\left(x_{\varrho} T_{\mu \sigma}(x)-x_{\sigma} T_{\mu \varrho}(x)\right) .
$$

${ }^{8}$ In massive current algebras the stress-tensor can be expressed in terms of the currents but we will not need such a relation 

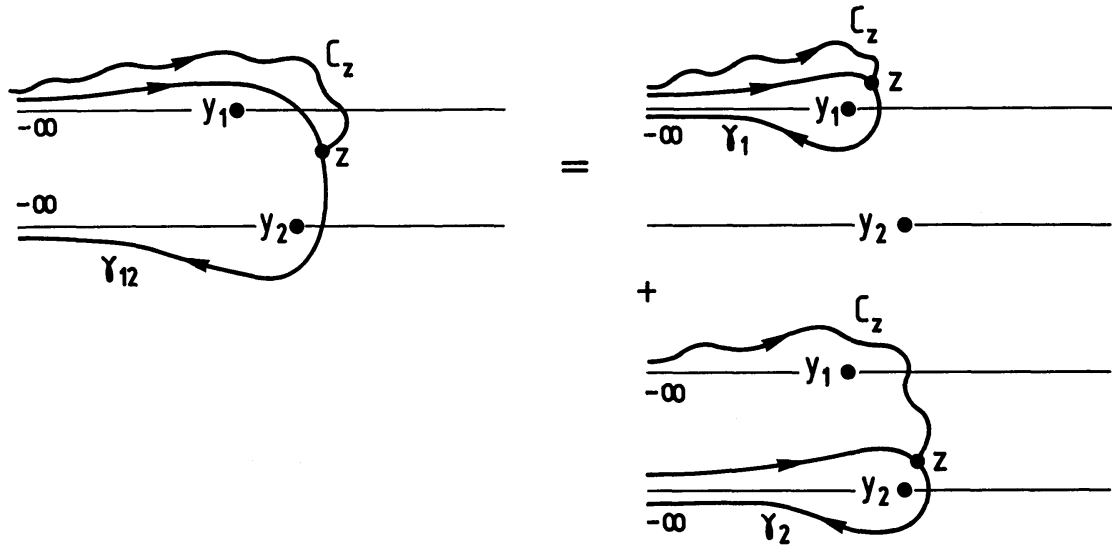

Fig. 6. Decomposition of the contours used in the proof of the comultiplication (21)

On the local fields $L$ acts as a commutator:

$$
L(\Phi(y))=L \Phi(y)-\Phi(y) L .
$$

The currents $J_{u}^{a}(x)$, which are one-forms, transform covariantly under Lorentz boost:

$$
L\left(J_{\mu}^{a}(x)\right)=\frac{1}{2} \varepsilon_{\rho \sigma}\left(x^{\varrho} \partial^{\sigma}-x^{\sigma} \partial^{\varrho}\right) J_{\mu}^{a}(x)+\varepsilon_{\mu \sigma} J_{\sigma}^{a}(x) .
$$

Hence $J_{+}^{a}(x)$ have Lorentz spin \pm 1 .

The (non-local) conserved charges satisfy the following algebraic relations:

$$
\begin{aligned}
{\left[Q_{0}^{a}, Q_{0}^{b}\right] } & =f^{a b c} Q_{0}^{c}, \\
{\left[Q_{0}^{a}, Q_{1}^{b}\right] } & =f^{a b c} Q_{1}^{c}, \\
{\left[L, Q_{0}^{a}\right] } & =0, \\
{\left[L, Q_{1}^{a}\right] } & =-\frac{C_{A d j}}{i 4 \pi} Q_{0}^{a},
\end{aligned}
$$

where $C_{A d j}$ is the Casimir of $\mathscr{G}$ in the adjoint representation in the normalization defined by Eq. (27a). This normalization corresponds to $\lambda=\frac{C_{A d j}}{i 2 \pi}$ in Eq. (10).

The relations (27) are part of the defining relations of the semi-direct product of the Yangians $Y(\mathscr{G})$ by the Poincaré algebra. Only the Serre relations are missing. (They are more difficult to prove because they involve commutation relations between the non-local charges.) Moreover the comultiplications (22b) and (23b) are the comultiplications in $Y(\mathscr{G})$. [See the appendix for more details on $Y(\mathscr{G})$.]

The normalization coefficient in Eq. (27d) is not arbitrary. It is the normalization which ensures the crossing symmetry of the $S$-matrices.

The two first relations are easily proved because, as usual, the commutation relations follow from the OPE's. The only delicate point is to check that the relation (27a) effectively corresponds to the normalization $\lambda=\frac{C_{A d j}}{i 2 \pi}$. This follows from the OPE's of the currents using $\lim _{\varepsilon \rightarrow 0^{+}} \frac{i \varepsilon}{x^{2}+\varepsilon^{2}}=i \pi \delta(x)$. The relation (27c) follows from Eq. (26). 


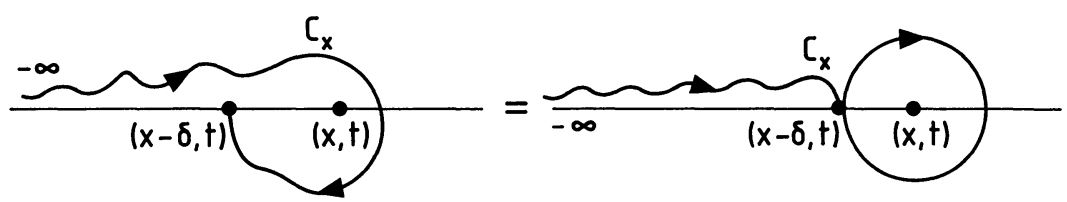

Fig. 7. Rotation of $2 \pi$ of the non-local currents

The relation (27d) is more interesting. A way to prove it could be to use the commutation relations and the OPE's described above, but there is a more geometrical way of doing it. It consists in imposing a Lorentz boost $\mathscr{R}_{2 \pi}$ of angle $(i 2 \pi)$ to the non-local currents $J^{(1) a}(x, t)$. It is a rotation of $(2 \pi)$ in the Euclidean plane. Because the currents $J_{\mu}^{(1) a}(x, t)$ are non-local this transformation does not act trivially on them: the string $\mathscr{C}_{x}$ winds around the point $x$ as in Fig. 7. By decomposing, as it is shown in Fig. 7, this windy contour into the sum of a contour from $-\infty$ to $x$ plus a small contour surrounding $x$ we obtain:

$$
\mathscr{R}_{2 \pi} J_{\mu}^{(1) a}(x, t) \mathscr{R}_{2 \pi}^{-1}=J_{\mu}^{(1) a}(x, t)-\frac{1}{2} f^{a b c} Q_{0}^{c}\left(J_{\mu}^{b}(x, t)\right) .
$$

Integrating the time-component of Eq. (28) over an equal-time slice gives

$$
\mathscr{R}_{2 \pi} Q_{1}^{a} \mathscr{R}_{2 \pi}^{-1}=Q_{1}^{a}-\frac{1}{2} C_{\text {Adj }} Q_{0}^{a} .
$$

in agreement with the relation $(27 \mathrm{~d})$ because $\mathscr{R}_{2 \pi}=\exp (i 2 \pi L)$.

We now illustrate these commutation relations by checking them on the primary fields. A primary field $\Phi_{\Lambda}(y, t)$, which we suppose to be valued in the representation $\Lambda$ of $\mathscr{G}$, is defined by its OPE with the currents $J_{\mu}^{a}(x, t)$ : a field $\Phi_{\Lambda}(y, t)$ is said to be primary if it is local with respect to $J_{\mu}^{a}(x, t)$ and if it satisfies the following OPE's:

$$
J_{\mu}^{a}(x) \Phi_{\Lambda}(0)=\frac{i}{2 \pi} \frac{x_{\mu}}{x^{2}}\left(T^{a} \Phi_{\Lambda}\right)(0)+\mathcal{O}\left(|x|^{-0}\right)
$$

The equal-time commutation relations between the currents $J_{\mu}^{a}$ and the field $\Phi_{\Lambda}$ can be deduced from the OPE's (30):

$$
\begin{gathered}
{\left[J_{0}^{a}(x, t), \Phi_{\Lambda}(y, t)\right]=\delta(x-y)\left(T^{a} \Phi_{\Lambda}\right)(y, t),} \\
Q_{0}^{a}\left(\Phi_{\Lambda}(y, t)\right)=\left(T^{a} \Phi_{\Lambda}(y, t)\right) .
\end{gathered}
$$

Here $T^{a}$ denote the matrices representing $\mathscr{G}$. Equation (30) also implies that $\Phi_{\Lambda}(y, t)$ commutes with $J_{1}^{a}(x, t)$.

The action of $Q_{1}^{a}$ on $\Phi_{\Lambda}(y, t)$ is deduced from Eqs. (21) and (31):

$$
Q_{1}^{a}\left(\Phi_{\Lambda}(y, t)\right)=\left[Q_{1}^{a}, \Phi_{\Lambda}(y, t)\right]+\frac{1}{2} f^{a b c}\left(T^{b} \Phi_{\Lambda}(y, t)\right) Q_{0}^{c}
$$

with

$$
\left[Q_{1}^{a}, \Phi_{\Lambda}(y, t)\right]=\frac{1}{2} f^{a b c}\left(\int_{-\infty}^{0^{-}} d x-\int_{0^{+}}^{+\infty} d x\right) J_{0}^{b}(y-x, t)\left(T^{c} \Phi_{\Lambda}\right)(y, t) .
$$

If we act with the Lorentz boost $L$ we have:

$$
\begin{aligned}
L\left(Q_{1}^{a}\left(\Phi_{\Lambda}(y, t)\right)=\right. & Q_{1}^{a}\left(L\left(\Phi_{\Lambda}(y, t)\right)\right) \\
& +\frac{1}{2} f^{a b c}\left(\int_{-\infty}^{0^{-}} d x-\int_{0^{+}}^{+\infty} d x\right)\left[L, J_{0}^{b}(y-x, t)\right]\left(T^{c} \Phi_{\Lambda}\right)(y, t) .
\end{aligned}
$$


Using Eq. (26) and integrating by part gives:

$$
\begin{aligned}
L\left(Q_{1}^{a}\left(\Phi_{\Lambda}(y, t)\right)=\right. & Q_{1}^{a}\left(L\left(\Phi_{\Lambda}(y, t)\right)\right) \\
& +\frac{1}{2} f^{a b c} \lim _{\delta \rightarrow 0^{+}}\left[\delta\left(J_{1}^{b}(y-\delta, t)-J_{1}^{b}(y+\delta, t)\right)\right]\left(T^{c} \Phi_{\Lambda}\right)(y, t) .
\end{aligned}
$$

Finally using the defining OPE's (30) for the primary fields we find:

$$
L\left(Q_{1}^{a}\left(\Phi_{\Lambda}(y, t)\right)=Q_{1}^{a}\left(L\left(\Phi_{\Lambda}(y, t)\right)-\frac{C_{a d j}}{i 4 \pi}\left(T^{a} \Phi_{\Lambda}(y, t)\right) .\right.\right.
$$

Equation (35) proves the commutation relation (27d) on the primary fields.

\section{2h) Action on the Asymptotic States}

We now describe how we get non-perturbative results on the $S$-matrices by looking at the action of the non-local charges on the asymptotic states. The constraints on the $S$-matrices we obtain arise by writing the commutation of the $S$-matrices with the non-local conserved charges. In the cases we are dealing with, these commutation relations will simply be the exchange algebras for the Yangians $Y(\mathscr{G})$.

The constructions explained in Sects. (2a) through $(2 \mathrm{~g})$ are model-independent. Different models are distinguished by their field content and spectrum of massive particles. According to our hypothesis, the fields are in one-to-one correspondence with fields in the conformal WZW theory. Thus the allowed representations of the massive current algebras are in correspondence with the integrable highest weight representations of $\mathscr{G}^{(1)}$ at level $K$. This also implies that the possible Lorentz spin of the particles corresponds to the conformal dimensions of these conformal fields. However not all the fields in the massive theory create asymptotic particles and not all the fields which create particles are affine primaries. Which fields do create particles is a question that cannot be answered by the formalism presented here. However from results of the representation theory of the Yangians $Y(\mathscr{G})$ [11], we know that the fields which create asymptotic particles in the fundamental representations $\Lambda_{i}$ of $\mathscr{G}$ are primaries only if the Kac label [12] of $\Lambda_{i}$ is one. For the level $K=1$, based on known results of the Gross-Neveu models, we expect the massive particles to be associated to the fundamental representations. For higher $K$ the $S U(2)$ models have been solved [8] and the conjectured spectrum of massive particles suggests that the particles are still associated with the fundamental representations, however with the distinction that they are now associated with fields that intertwine the fundamental representations with the other integrable highest weight representations.

The way to deduce the action of $Q_{1}^{a}$ on the asymptotic states is clear. It is enough to compute the action on the one-particle states because we already know the comultiplication. First we identify the (asymptotic) fields, called $\Phi_{A}$, which

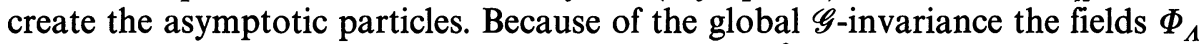
belong to some representation $\Lambda$ of the Lie algebra $\mathscr{G}^{9}$. The antiparticles belong to the conjugated representation $\Lambda^{*}$ and they are asymptotically created by the fields $\Phi_{\Lambda^{*}}$. Secondly because the local conserved currents $J_{\mu}^{a}(x)$ appear in the OPE's

9 The fields $\Phi_{A}$ may carry other quantum numbers as they do in the FSSG models [8] 
between $\Phi_{\Lambda}$ and $\Phi_{\Lambda^{*}}$ they can be written as (generalized) normal ordered products of the two conjugated fields $\Phi_{\Lambda}$ and $\Phi_{\Lambda^{*}}$. Finally once we have the expression of the currents in terms of $\Phi_{\Lambda}$ we can plug it in the definition (11) of $J^{(1)}(x)$ and use it to compute the action of $Q_{1}^{a}$.

We illustrate this general strategy by discussing simple examples. We will consider the cases in which the asymptotic fields are fermions. The $S O(N)$ GrossNeveu models are concrete examples corresponding to this hypothesis: they are equivalent to the $S O(N)$ massive current algebras at level $K=1$. In the $S O(N)$ Gross-Neveu models the asymptotic fermions are Majorana fermions taking values in the vector representation of $S O(N)$. We choose these examples mainly in order to simplify the exposition.

Let us now do the fermionic computation ${ }^{10}$. We denote by $\Psi^{k}(x), k=1, \ldots, N$, the asymptotic Majorana fermions and by $\psi^{k}(x)$ and $\bar{\psi}^{k}(x)$ their components: $\Psi^{k}=\left(\psi^{k}, i \bar{\psi}^{k}\right)^{t 11}$. Asymptotically when $t \rightarrow-\infty$ the in-fermions are free and the fields $\Psi^{k}(x)$ can be decomposed as follows:

with

$$
\begin{aligned}
& \psi^{k}(x)=\sqrt{m} \int \frac{d \theta}{4 \pi} e^{\theta / 2}\left[b^{k \dagger}(\theta) e^{-i(p, x)}+b^{k}(\theta) e^{+i(p, x)}\right] \\
& \bar{\psi}^{k}(x)=\sqrt{m} \int \frac{d \theta}{4 \pi} \frac{e^{-\theta / 2}}{i}\left[b^{k \dagger}(\theta) e^{-i(p, x)}-b^{k}(\theta) e^{+i(p, x)}\right]
\end{aligned}
$$

$$
\left\{b^{k}(\theta), b^{n \dagger}\left(\theta^{\prime}\right)\right\}=4 \pi \delta^{k n} \delta\left(\theta-\theta^{\prime}\right),
$$

where $(p, x)=\varepsilon t-p x$ and $(\varepsilon=m \operatorname{ch} \theta, p=m s h \theta)$ are the energy and the momentum of the fermions of mass $m$ and rapidity $\theta$. The asymptotic in-fermions satisfy the Dirac equation $(i \not \partial-m) \Psi^{k}=0$. A similar decomposition holds for the out-fermions (when $t \rightarrow+\infty$ ). In order to avoid cumbersome notations we will deal only with the infermions and will suppress the "in" indices.

The currents $J^{a}(x)$ are bilinear in the fermions:

$$
J_{\mu}^{a}(x)=\frac{1}{2}: \bar{\Psi}(x) \gamma_{\mu} T^{a} \Psi(x): .
$$

Here the double dots denote the fermionic normal order. The $T^{a}$ s form the $N$ dimensional representation of $S O(N): T^{a} \equiv T^{k l}$ with $\left(T^{k l}\right)=\delta^{k m} \delta^{l n}-\delta^{l m} \delta^{k n}$. We have normalized the currents such that the fermions are primaries in the sense of Eq. (30). In particular we have:

$$
Q_{0}^{k l}(\Psi(x))=\left(T^{k l} \Psi(x)\right)
$$

This normalization corresponds to $\lambda=\frac{C_{A d j}}{i 2 \pi}$ in Eq. (10). In our normalization for the $T^{k l}$ 's, which is not the standard one, $C_{A d j}=4 \mathrm{~h}^{*}$ with $h^{*}$ the dual Coxeter number of $S O(N)$. Also the mass scale $M$ in Eq.(10) is related to the mass $m$ of the fermions by $M=\frac{1}{2} m e^{C}$ with $C$ the Euler's constant.

Because the vacuum is annihilated by all the charges the value of the commutator (32b) on the vacuum is the value of $Q_{1}^{a}$ on the one-particle states.

${ }^{10}$ I thank A. Leclair for suggesting to me this simple way of doing the computation

${ }_{11}$ We use the following conventions for the Dirac matrices: $\left\{\gamma^{\mu}, \gamma^{\nu}\right\}=2 \eta^{\mu v}$ with $\gamma^{0}=\left(\begin{array}{ll}0 & 1 \\ 1 & 0\end{array}\right)$ and $\gamma^{1}=\left(\begin{array}{cc}0 & -1 \\ 1 & 0\end{array}\right)$ 
Using the Wick theorem we find:

$$
\left[Q_{1}^{a}, \Psi(y)\right]|0\rangle=\frac{C_{A d j}}{4} \int_{-\infty}^{+\infty} d x \varepsilon(x) \Delta(x) \gamma_{0}\left(m+i \gamma^{1} \partial_{x^{1}}\right)\left(T^{a} \Psi(y-x)\right)|0\rangle
$$

with $\varepsilon(x)=\operatorname{sign}(x)$ and $\Delta(x)=\frac{1}{2 \pi} K_{0}(m|x|)$, where $K_{0}$ is the $0^{\text {th }}$ Bessel function [19].

Using the Fourier decomposition (36a) we obtain the formula for the action on one-fermion states ${ }^{12}$ :

$$
Q_{1}^{a}\left(b^{\dagger}(\theta)|0\rangle\right)=-\frac{\theta C_{A d j}}{i 4 \pi}\left(T^{a} b^{\dagger}(\theta)|0\rangle\right) .
$$

The action on many-fermion states follows from the comultiplication (23b) and Eq. (38).

In the $S O(N)$ Gross-Neveu models all the previous computations are summarized as follows:

$$
\begin{aligned}
& Q_{0}^{k l}=T^{k l}, \\
& Q_{1}^{k l}=-\frac{\theta(N-2)}{i \pi}\left(T^{k l}\right), \\
& \Delta Q_{1}^{k l}=Q_{1}^{k l} \otimes 1+1 \otimes Q_{1}^{k l}-\sum_{n}\left(T^{k n} \otimes T^{n l}-T^{l n} \otimes T^{n k}\right),
\end{aligned}
$$

where the $T^{k l}$ s form the vector representation $\square$ of $S O(N):\left(T^{k l}\right)^{m n}=\delta^{k m} \delta^{l n}-\delta^{l m} \delta^{k n}$. The charges $Q_{0}^{a}$ and $Q_{1}^{a}$ defined in Eq. (41) satisfy the algebra (27) because on-shell the boost operator $L$ acts as $\frac{\partial}{\partial \theta}$. They define an irreducible representation of the $S O(N)$-Yangians in the vector representation of $S O(N)$. Equation (41c) is the comultiplication in $Y((S O(N))$. (See the appendix for more details.)

Let us now discuss the implications on the scattering of two fermions of rapidities $\theta_{1}$ and $\theta_{2}$. Denote by $S\left(\theta_{12}\right), \theta_{12}=\theta_{1}-\theta_{2}$, the $S$-matrix of this process. $S(\theta)$ acts from $\square \otimes \square$ into itself. As an $S O(N)$ representation the tensor product $\square \otimes \square$ decomposes into $(\theta+\varangle+\bullet)$. We denote by $P_{-}, P_{+}$, and $P_{0}$ the respective projectors. By $S O(N)$-invariance, $S(\theta)$ decomposes on these projectors:

$$
S(\theta)=\sigma_{+}(\theta) P_{+}+\sigma_{-}(\theta) P_{-}+\sigma_{0}(\theta) P_{0},
$$

where $\sigma_{n}(\theta)$ are scattering amplitudes.

The non-local charges $Q_{1}^{k l}$ are conserved. Therefore they commute with the $S$ matrix. For the two-fermion scattering it implies:

$$
\left(\Delta_{2 ; 1} Q_{1}^{k l}\right) \hat{S}\left(\theta_{12}\right)=\hat{S}\left(\theta_{12}\right)\left(\Delta_{1 ; 2} Q_{1}^{k l}\right),
$$

where $\hat{S}(\theta)=P S(\theta)$ with $P$ the flip operator: $P(x \otimes y)=y \otimes x$. The comultiplication is defined in Eq. (41c). The indices 1 and 2 refer to the rapidities $\theta_{1}$ and $\theta_{2}$. Equation (43) is the exchange relations for $Y(S O(N))$ in the vector representation. Equation (43) leads to algebraic relations between the scattering amplitudes:

$$
\frac{\sigma_{-}(\theta)}{\sigma_{+}(\theta)}=\frac{\theta(N-2)+i 2 \pi}{\theta(N-2)-i 2 \pi} ; \quad \frac{\sigma_{0}(\theta)}{\sigma_{-}(\theta)}=\frac{\theta+i \pi}{\theta-i \pi} .
$$

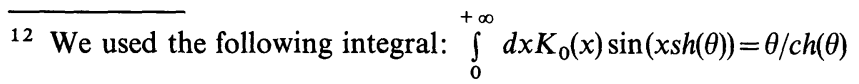


Equations (44) determine $S(\theta)$ up to an overall function which could be fixed by closing the bootstrap program [20]. Note that the $S$-matrix (42) is (up to an overall scalar function) a rational solution of the Yang-Baxter equations. This could have been guessed from the beginning since the non-local conserved charges have spin zero.

Hence we have been able to deduce non-perturbative results for the $S$-matrix by using the exact non-local conserved charges.

\section{Conclusion}

For the readers who have been courageous enough to go all through the eight steps of Sect. 2 it is now clear that non-local conserved currents can be exactly defined. They give non-perturbative results in massive two dimensional quantum field theories. Surely they will play a master role in the algebraic framework for massive QFT which will parallel the algebraic approach to CFT set up these last few years.

To find non-perturbative results for the $S$-matrices it is enough to have at our disposal only finite dimensional representations of the algebra of the non-local conserved charges. This point was illustrated in Sect. $2 \mathrm{~h}$. For the correlation functions the existence of the conversed charges $Q_{k}^{a}$ implies Ward identities. These can be written as in [21]:

$$
\Delta^{(N)}\left(Q_{k}^{a}\right)\left\langle\Phi_{1}\left(y_{1}\right) \ldots \Phi_{N}\left(y_{N}\right)\right\rangle=0,
$$

where $\Delta^{(N)}$ is the $N^{\text {th }}$ comultiplication. In order for the Ward identities to determine the correlation functions the algebra of the conserved charges should (presumably) possess only infinite dimensional representations. Thus in order to formulate an algebraic approach to massive QFT's, alternative to the quantum inverse scattering methods, we should (presumably) look for extensions of the algebra of the non-local charges (extensions of the Yangians in our cases) which admit only infinite dimensional representations.

\section{Appendix A. Yangians $Y(\mathscr{G})$}

In this appendix we present a very short review of the basic definitions and theorems of the theory of the Yangians. We follow Drinfel'd [11]. We denote by $Y(\mathscr{G})$ the Yangian over the semi-simple Lie algebra $\mathscr{G}$. It is defined as follows.

Definition. The Yangian $Y(\mathscr{G})$ is the associative algebra with unity generated by the elements $J_{0}^{a}$ and $J_{1}^{a}$ with the defining relations:

$$
\begin{gathered}
{\left[J_{0}^{a}, J_{0}^{b}\right]=f^{a b c} J_{0}^{c},} \\
{\left[J_{0}^{a}, J_{1}^{b}\right]=f^{a b c} J_{1}^{c},} \\
{\left[J_{1}^{a},\left[J_{1}^{b}, J_{0}^{c}\right]\right]-\left[J_{0}^{a},\left[J_{1}^{b}, J_{1}^{c}\right]\right]=A_{d e f}^{a b c}\left\{J_{0}^{d}, J_{0}^{e}, J_{0}^{f}\right\},} \\
{\left[\left[J_{1}^{a}, J_{1}^{b}\right],\left[J_{0}^{c}, J_{1}^{d}\right]\right]+\left[\left[J_{1}^{c}, J_{1}^{d}\right],\left[J_{0}^{a}, J_{1}^{b}\right]\right]} \\
=\left(A_{l m n}^{a b k} f^{c d k}+A_{l m n}^{c d k} f^{a b k}\right)\left\{J_{0}^{l}, J_{0}^{m}, J_{1}^{n}\right\},
\end{gathered}
$$

where $f^{a b c}$ are the structure constants of $\mathscr{G}, A_{d e f}^{a b c}=\frac{1}{24} f^{a d k} f^{b e l} f^{c f m} f_{k l m}$ and $\left\{x_{1}, x_{2}, x_{3}\right\}=\sum_{i \neq j \neq k} x_{i} x_{j} x_{k}$.

We have the following property. 
Proposition. $Y(\mathscr{G})$ is a Hopf algebra with comultiplication $\Delta$ :

$$
\begin{gathered}
\Delta\left(J_{0}^{a}\right)=J_{0}^{a} \otimes 1+1 \otimes J_{0}^{a}, \\
\Delta\left(J_{1}^{a}\right)=J_{1}^{a} \otimes 1+1 \otimes J_{1}^{a}-\frac{1}{2} f^{a b c} J_{0}^{b} \otimes J_{0}^{c} .
\end{gathered}
$$

Moreover $Y(\mathscr{G})$ admits the following automorphism: $T_{v}: Y(\mathscr{G}) \rightarrow Y(\mathscr{G})$ such that $T_{v}\left(J_{0}^{a}\right)=J_{0}^{a}$ and $T_{v}\left(J_{1}^{a}\right)=J_{1}^{a}+v J_{0}^{a}$. This automorphism is called the "evaluation" automorphism. The interest of this automorphism resides in the following theorem.

Theorem. There is a unique formal series $\mathscr{R}(v)=1+\sum_{k} \mathscr{R}_{k} v^{-k}$ in $Y(\mathscr{G}) \otimes Y(\mathscr{G})$ such that:

$$
\begin{gathered}
(1 \otimes \Delta) \mathscr{R}(v)=\mathscr{R}_{12}(v) \mathscr{R}_{23}(v), \\
\mathscr{R}(v)\left(\left(T_{v} \otimes 1\right) \Delta(x)\right)=\left(\left(T_{v} \otimes 1\right) P \Delta(x)\right) \mathscr{R}(v) ; \quad \text { for } \quad x \in Y(\mathscr{G}) .
\end{gathered}
$$

Equation (A.3a) implies the Yang-Baxter equation for $\mathscr{R}(v)$. Equation (A.3b) defines the exchange algebra for $Y(\mathscr{G})$. It gives rise to algebraic equations which can be used to determine $\mathscr{R}(v)$.

Acknowledgements. It is a pleasure to thank G. Felder and A. Leclair without whom this work would not have been done. I thank D. Altschuler, A. Belavin, N. Reshetikhin, and J.-B. Zuber for discussions.

Note Added in Proof. After having submitted the paper we learned that non-local charges in integrable two-dimensional quantum field theories have also been studied by the authors of [22]. They also noted, as we did in Eq. (43), the relation between the conservation law of the non-local conserved charges and the exchange relations of the algebraic Bethe ansatz. But their approach was different from ours because in order to define (on-shell) the non-local charges they supposed that the $S$-matrix and the co-multiplication of the non-local charges are known.

\section{References}

1. Belavin, A., Polyakov, A., Zamolodchikov, A.: Infinite dimensional symmetry in twodimensional quantum field theory. Nucl. Phys. B 241, 333-380 (1984)

2. Conformal invariance and applications to statistical mechanics. Itzykson, C., Saleur, H., Zuber, J.-B. (eds.) Singapore: World Scientific 1988

3. Zamolodchikov, A.B.: Integrable field theory from conformal field theory. Adv. Studies Pure Math. 19, 641-674 (1989)

Zamolodchikov, A.B.: Integrals of motion in scaling 3-state Potts model field theory. Int. J. Mod. Phys. A 3, 743-750 (1988)

Zamolodchikov, A.B.: Integrals of motion and $S$-matrix of the (scaled) $T=T_{C}$ Ising models with magnetic field. Int. J. Mod. Phys. A 4, 4235 (1989)

4. Leclair, A.: Restricted sine-Gordon theory and the minimal conformal series. Phys. Lett. 320B, 103 (1989)

5. Smirnov, F.A.: The perturbed $c<1$ conformal field theories as reductions of sine-Gordon models. Int. J. Mod. Phys. A 4, 4213 (1989)

6. Eguchi, T., Yang, S.K.: Deformations of conformal field theories and soliton equations. Phys. Lett. 235B, 373 (1989)

7. Kupershmidt, B.A., Mathieu, P.: Quantum Korteweg-deVries equations and perturbed conformal field theories. Phys. Lett. 227B, 245-250 (1989)

8. Bernard, D., Leclair, A.: The fractional supersymmetric sine-Gordon models. Phys. Lett. B 247, 309 (1990) 
9. Zamolodchikov, A.B.: Preprint, Sept. 1989 (unpublished)

10. Bernard, D., Leclair, A.: Residual quantum symmetry in the restricted sine-Gordon theories. Nucl. Phys. B 340, 721 (1990)

Ahn, C., Bernard, D., Leclair, A.: Fractional Supersymmetries in perturbed coset CFT's and integrable soliton theory. Nucl. Phys. B 346, 409 (1990)

11. Drinfel'd, V.G.: Hopf algebras and the quantum Yang-Baxter equation. Sov. Math. Dokl. 32, 254-258 (1985)

12. Kac, V.G.: Infinite dimensional Lie algebras. Cambridge: Cambridge University Press 1985

13. Knizhnik, V.G., Zamolodchikov, A.B.: Current algebras and Wess-Zumino models in two dimensions. Nucl. Phys. B 247, 83-103 (1984)

14. Maillet, J.-M.: New integrable canonical structures in two-dimensional models. Nucl. Phys. B 269, 54-76 (1986) and Hamiltonian structures for integrable classical theories from graded Kac-Moody algebras. Phys. Lett. 167 B, 401-405 (1986)

15. Lüscher, M., Pohlmeyer, K.: Scattering of massless lumps and non-local charges in the twodimensional classical non-linear $\sigma$-models. Nucl. Phys. B 137, 46-54 (1978)

16. Brezin, E., et al.: Remarks about the existence of non-local charges in two-dimensional models. Phys. Lett. 82B, 442-444 (1979)

17. Lüscher, M.: Quantum non-local charges and absence of particle production in the twodimensional non-linear $\sigma$-models. Nucl. Phys. B 135, 1-19 (1978)

18. Coleman, S., Mandula, J.: All possible symmetries of the $S$-matrix. Phys. Rev. 159, 1251 (1967)

19. Gradshteyn, I.S., Ryzhik, I.W.: Table of integrals, series and products. New York: Academic Press 1965

20. Zamolodchikov, A.B., Zamolodchikov, A.B.: Factorized $S$-matrices in two dimensions as the exact solutions of certain relativistic quantum field theories. Ann. Phys. 120, 253 (1979)

21. Bernard, D., Leclair, A.: $Q$-deformation of the $S U(1,1)$ conformal ward identities and $Q$ string. Phys. Lett. 227B, 417-423 (1989)

22. De Vega, H., Eichenherr, H., Maillet, J.M.: Yang-Baxter algebras of monodromy matrices in integrable quantum field theories. Nucl. Phys. B 240[FS12], 377-399 (1984) and Classical and quantum algebras of non-local charges in $\sigma$-models. Commun. Math. Phys. 92, 507-524 (1984)

Communicated by K. Gawedzki 